\title{
Biomechanical implications of mild leg length inequality
}

\author{
S. T. McCaw, BPHE, PhD ${ }^{1}$ and B. T. Bates, BEng, PhD $^{2}$ \\ ${ }^{1}$ Department of Health, Physical Education, Recreation and Dance, Illinois State University, USA \\ ${ }^{2}$ Biomechanics/Sports Medicine Laboratory, University of Oregon, Eugene, Oregon, USA
}

\begin{abstract}
The effect of mild leg length inequality (lower extremity length difference less than $3 \mathrm{~cm}$ ) on posture and gait has been the source of much controversy. Many opinions have been expressed both for and against the need for intervention to reduce the magnitude of the discrepancy. This paper emphasizes the need for accurate and reliable assessment of leg length differences using a clinically functional radiographic technique, and reviews the biomechanical implications of leg length inequality as related to the development of stress fractures, low back pain and osteoarthritis.
\end{abstract}

Keywords: Leg length inequality, gait, stress fractures, low back pain, osteoarthritis.

Bilateral asymmetries have been documented for a variety of anatomical characteristics ${ }^{1-3}$. These differences are thought to develop from both genetic and environmental factors influencing the ontogenesis of the individual.

A bilateral asymmetry in lower limb lengths is called leg length inequality (LLI), or anisomelia. While the possible negative cosmetic effects of LLI are obvious, the biomechanical implications of mild LLI (difference $<3 \mathrm{~cm}$ ) remain a source of speculation based on limited research. The purpose of this paper is to review the literature pertaining to mild LLI to identify possible links between the asymmetry and orthopaedic disorders.

\section{Definition of leg length inequality}

A differentiation must be made between the anatomical, or strucurally short leg, and the functional short leg 4 .

A functional short leg occurs secondary to a rotated pelvis caused by joint contractures and/or axial malalignments, including scoliosis ${ }^{5}$. The foot on the 'short limb' side is externally rotated, the heel is in the valgus position, and the longitudinal arch of the foot is collapsed. The posterior iliac spine is high on the 'short leg' side, while the anterior iliac spine is higher on the 'long leg' side.

Address for correspondence: Steven T. McCaw, PhD, Department of HPERD, Illinois State University, Normal, Illinois 61761, USA

(C) 1991 Butterworth-Heinemann Ltd.

0306-3674/91/010010-04
An anatomical short leg occurs when there is an actual length difference in the bony components of the lower limb. A structurally short limb is often compensated for by a functional adaptation on the long leg side, including ankle pronation ${ }^{6}$. The anterior and posterior iliac spines are lower on the side of the short leg, unless foot positioning compensates for the difference.

A third category, environmental LLI, is especially relevant to road runners. The drainage slope built into roads imposes a height difference between the limb on the curb side and that on the midline side of the road. In runners with an existing LLI, this environmental effect can accentuate the difference if the short leg is closest to the curb, and attenuate, eliminate or even reverse the inequality if the long leg is on the curb side ${ }^{7}$.

\section{Techniques for assessing lower limb length}

Both radiographic and tape-measured methods are used to assess the length of the lower limbs. The magnitude of the absolute error inherent to the measurement technique provides an operational definition of $\mathrm{LLI}^{8}$. The reported validity and intraand inter-tester reliability coefficients of tapemeasured methods are consistently less than radiographic measures ${ }^{9,10}$. Tape-measurement inaccuracy stems from its reliance on bony prominences, primarily the anterior superior iliac spine and the medial malleolus. Tape-measured leg length differences of less than $1.27 \mathrm{~cm}$ (one-half inch) may be unreliable $^{11}$. Although prone to measurement error, tape-measurements of LLI are frequently used in routine orthopaedic examinations because they are easy to administer and cost very little.

Radiographic techniques provide the measurement accuracy and reliability necessary for research and clinical practice. Radiographs have not received widespread use due to ethical objections to radiation exposure and their prohibitive cost. However, the development and validation of femoral head radiographs has reduced both the radiation exposure and the cost of accurately assessing LLI.

To obtain a femoral head radiograph, the subject stands on a level platform in either an anteroposterior or posteroanterior projection, with the pelvis as close 
as possible to the film (the approximate hip to film distance is $18-20 \mathrm{~cm})$. The tube is set at the estimated height of the subject's greater trochanter, plus $2.54 \mathrm{~cm}$. The subject assumes a 'parallelogram posture $^{\prime 12}$ in which the feet are aligned parallel, with the first metatarsals separated by the mean femoral head centre distance of $18.6 \mathrm{~cm}^{13}$ and the lower extremity joints in the anatomical position. The parallelogram consists of the two lower extremities, the intermetatarsal distance, and the femoral head centres. In the absence of gross errors of positioning and/or tube placement, the method provides an accurate measure of the relative magnitude of the discrepancy between limb lengths, and not the difference between the absolute length of the limbs. Using this technique, the mean error between repeated measurements of the same subjects on different days was $<2 \mathrm{~mm}$. Gofton and Trueman ${ }^{12}$ had a mean of $1.5 \mathrm{~mm}$; Friberg et al. ${ }^{14}$ had a mean of $0.6 \mathrm{~mm}$ and a range of $0-2 \mathrm{~mm}$.

Restricting measurements to the pelvic region reduces the radiated area of the body and the total radiation dose. Using a $1.8 \mathrm{~mm}$ thick, $12 \times 20 \mathrm{~cm}$ gonadal shield limits the mean doses to gonads and bone marrow to 11.4 milliRads $(\mathrm{mR})$ and $13.6 \mathrm{mR}$, respectively $\left(1.14 \times 10^{-9}\right.$ and $1.36 \times 10^{-9} \mathrm{~Gy}$ respectively), which is less than the radiation doses associated with conventional radiographic techniques of LLI. Friberg et al. ${ }^{14}$ concluded that femoral head radiography can be regarded as risk free and suitable for screening studies.

\section{Biomechanical significance of the asymmetry}

Three categories of LLI have been identified based on the magnitude of the discrepancy ${ }^{15}$ : mild (differences $<3 \mathrm{~cm}$ ), moderate (differences $\geqslant 3$ and $\leqslant 6 \mathrm{~cm}$ ), and severe (differences $>6 \mathrm{~cm}$ ).

The definition of a biomechanically meaningful asymmetry differs amongst orthopaedic specialists. While structural deformity and functional disturbances have been unequivocally linked with moderate and severe discrepancies ${ }^{16}$, mild differences have not been convincingly linked with any specific pathology. A paucity of research focusing on mild LLI compounds the problem of designing intervention. Gofton $^{17}$ was critical of the available literature that did not identify a definitive link between mild LLI and specific injuires. He claimed that the use of tape-measured assessments of LLI introduced enough variability to mask existing relationships. The questions of when intervention is necessary and the optimal modality for correcting mild LLI remain controversial. The remainder of this review will focus on mild LLI since it is the most controversial classification.

Several authors ${ }^{18,19}$ consider the biomechanical disruption caused by mild LLI to be inconsequential and propose that postural adjustments compensate for the asymmetry. However, several authors ${ }^{4,15,20-22}$ concur that mild LLI may be deleterious if an individual participates regularly in tasks involving repetitive mechanical loading.

Gross $^{18}$ investigated the hypothesis that repetitive impulsive loading aggravates the effects of LLI. He hypothesized that the occurrence of the asymmetry would be lower in marathon runners than among the general population through attrition of runners with LLI. However, 34 of 35 runners participating in the study had unequal limb lengths, measured by radiography. He concluded that 'runners can function quite handsomely without equalization' of the asymmetry, in spite of the numerous unilateral injuries reported by the subjects.

Mild LLI has primarily been associated with three orthopaedic disorders: stress fractures, low back pain and osteoarthritis. The following sections provide a selected review of the literature relating mild LLI to each of these disorders.

\section{Stress fractures}

Stress fractures are focal structural weaknesses in bone developed during the osseous remodelling that occurs in response to repeated application of subthreshold stresses ${ }^{23}$. As energy is absorbed to attenuate the applied load, microfractures develop in the bone. Remodelling of the bone occurs as the microfractures heal. The microfracture/remodelling process is stable at subthreshold loads. If the threshold is exceeded, then the rate of new bone formation is slower than the rate of osteoclastic resorption and a stress fracture develops.

Matheson et al. ${ }^{23}$ reviewed the two most accepted theories of stress fracture development: (1) muscle weakness reduces the shock absorbing capability of the lower limb, causing an increase and redistribution of stress to the bone; and (2) the force of muscle pull on bone repetitively exceeds the threshold stress. It is plausible that a leg length inequality could contribute to either of these two aetiologies.

Two roles of the tibia include weight-bearing and muscle attachment. In a study of 130 military recruits, Friberg $^{21}$ reported that the majority of stress fractures in the weight-bearing bones (tibia and femur) occurred in the long limb. In normal stance, body-weight is evenly distributed between both lower limbs. In persons with LLI there is a tendency to shift weight-bearing towards the longer limb24. The effect of strenuous movement on weight distribution is not clear.

Individuals with LLI may pronate the foot on the side of the longer limb in an attempt to provide functional shortening of the longer $\operatorname{leg}^{6}$. Injury avoidance during gait could be compromised by the reduced range of pronation during contact. Muscular stress to the tibia could be increased by the enhanced eccentric activation of the tibialis anterior muscle required to resist calcaneal eversion as the joint nears the end of its range of movement. Both of these mechanisms could account for the pattern or stress fracture occurrences reported by Friberg ${ }^{21}$.

In summary, skeletal compensations to LLI may be associated with the development of stress fractures in the lower extremity. A better understanding of the compensatory skeletal realignment in response to LLI and its effect on bilateral loading of the limbs is needed to substantiate this association. 


\section{Low back pain}

Scoliosis is an orthopaedic ailment characterized by a lateral curvature of the spinal column. Two classifications of scoliosis are commonly used ${ }^{25}$. Functional scoliosis is a transient phenomenon in individuals standing with poor posture. Structural scoliosis is a permanent lateral bending of the spine, unaltered by muscle activity or postural adjustment.

The pelvic tilt common to LLI may invoke a functional scoliosis, concave to the side of the longer limb. Pelvic tilting helps maintain the line of the centre of gravity mediolaterally within the base of support. The degree of scoliosis is related to the magnitude of the LLI. The literature is unequivocal as to whether transient functional scoliosis will become structural over time ${ }^{26}$.

It has been hypothesized that LLI-induced scoliosis may be a causal factor in the development of non-specific low back pain and sciatica. Scoliotic subjects frequently suffer from sciatica on the concave side of the curved spine. In the scoliotic spine, the annulus of the intervertebral disc on the concave side of the spine is in compression, while that on the convex side is in tension. The compressed annulus can protrude out of the intervertebral space and impinge on the dorsal sensory nerve root.

Several investigators have evaluated the relationship between LLI and low back pain. Results have been unequivocal. Grundy and Roberts ${ }^{8}$ and Botte ${ }^{25}$ reported no link between the presence of LLI and low back pain, while Friberg ${ }^{27}$, Matheson et al. ${ }^{23}$ and Giles and Taylor $^{28}$ reported that low back pain was prevalent among those with LLI. Several investigators have reported success in reducing low back pain using orthotic lifts to equalize a $\mathrm{LLI}^{6,27,29}$.

The above-cited literature supports the hypothesis that LLI imposes a structural malalignment on the lower back, but does not unequivocally relate the asymmetry to back pain. More research investigating the link between LLI and low back pain is warranted by the findings.

\section{Osteoarthritis}

Osteoarthritis is a degenerative joint disease characterized by the thinning and eventual disappearance of articular cartilage. Articulating bone surfaces may become thickened, and the development of osteophytes projecting into the joint space is common. In advanced stages of the disease, the cartilage-free surfaces of the articulating bones may become severely deformed ${ }^{30}$.

Osteoarthritis has been categorized as primary (idiopathic) or secondary. Secondary osteoarthritis refers to cases where some antecedent event such as a fracture, childhood disease or skeletal abnormality is recognized as a predisposing factor. All other cases are considered idiopathic. Solomon ${ }^{31}$ questioned the existence of idiopathic osteoarthritis after identifying a predisposing abnormality in 300 of 327 cases of osteoarthritic hip. He proposed three pathogenic groups of secondary osteoarthritis: (1) failure of essentially normal cartilage subjected to abnormal or incongruous loading for long periods; (2) damaged or defective cartilage failing under normal conditions of loading; and (3) breakup of articular cartilage due to defective subchondral bone.

The pelvic tilt imposed by LLI may impose bilaterally unequal stresses in the hip and the knee joints during upright posture ${ }^{12,30}$. A tilted pelvis shifts the line of action of the centre of gravity away from the hip joint centre on the side of the long limb. The greater muscle activity necessary to compensate for the shift could increase the magnitude of the internal joint force. Pelvic tilt also reduces the contacting area of the articulating joint surfaces by disrupting normal skeletal alignment. These two effects, increased force and decreased articulating surface area, would result in increased stress on the cartilage and underlying bone, and represent a possible biomechanical precursor to osteoarthritis ${ }^{32}$.

During locomotion the lower extremity joint stresses are further increased by the muscle activity required to control the segments, by inertial forces developed by the moving segments, and by the impulsive force applied to the foot at ground contact. Although eccentric muscle activity and tissue compliance attenuate the impulsive force as it is transferred towards the head ${ }^{33}$, Voloshin and Wosk ${ }^{34}$ reported a decrease in the shock-attenuating capability of limbs affected with osteoarthritis. Reducing the dynamic stresses imposed on the limbs is believed to be a method of reducing the risk of degenerative disease.

Although evaluating asymmetry in lower extremity dynamics in the presence of LLI would increase our understanding of the biomechanical implications of LLI, there has been a paucity of research in this area. The pronated foot position used to compensate for LLI may reduce the impulse-attenuating capability of the long limb ${ }^{35}$. Vink and Huson ${ }^{36}$ reported that artificial LLI, created by wearing a single raised shoe, imposed minimal effects on pelvic kinematics and low back muscle activity during walking. If future research can indicate that LLI subjects one limb to pathogenic stresses, then the effect of equating limb lengths by using shoe raises is worth investigating, since it may be a very cost-effective method for preventing osteoarthritis.

\section{Acknowledgement}

Preparation of the review was supported in part by a Grant-in-Aid of Dissertation Research provided to Dr McCaw by the Foundation for Chiropractic Education and Research.

\section{References}

1 Singh I. Functional asymmetry in the lower limbs. Acta Anat 1970; 77: 131-8.

2 Baum I, Spencer AM. Limb dominance: its relationship to foot length. J Am Podiatry Assoc 1980; 70: 505-7.

3 Vagenas G, Hoshizaki TB. Optimization of an asymmetrical motor skill: the sprint start. Int J Sports Biomech 1986; 2: 29-40.

4 Subotnick SI. The short leg syndrome. J Am Podiatry Assoc 1976; 66: 720-3.

5 Eichler J. Methodological errors in documenting leg length and leg length discrepancies. In: Hungerford DS, ed. Leg length discrepancy, the injured knee Prog Orthop Surg. New York: Springer-Verlag, 1977: 29-39.

6 Langer S. Structural leg shortage: a case report. J Am Podiatry Assoc 1976; 66: 38-40. 
7 DuPlessis MP. Stress fractures and over-use syndromes (letter). S Afr Med J 1980; 58: 670.

8 Grundy PF, Roberts CJ. Does unequal leg length cause back pain? A case-control study. Lancet 1984; iv: 256-8.

9 Clarke GR. Unequal leg length: an accurate method of detection and some clinical results. Rheum Phys Med 1972; 11: 385-90.

10 DeBoer KF, Harmon RO, Savoie S, Tuttle CD. Inter- and intra-examiner reliability of leg-length differential measurements: a preliminary study. J Manipulative Physiol Ther 1983; 6 : 61-6.

11 Beal MC. The short leg problem. J Am Osteopath Assoc 1977; 76: 745-51.

12 Gofton JP, Trueman GE. Studies in osteoarthritis of the hip. Part II: Osteoarthritis of the hip and leg-length inequality. Can Med Assoc J 1971; 104: 791-9.

13 Ireland I, Kessel L. Hip adduction/abduction deformity and apparent leg-length inequality. Clin Orthop 1980; 153: 156-7.

14 Friberg O, Koivisto E, Wegelius C. A radiographic method for measurement of leg length inequality. Diagn Imag Clin Med 1985; 54: 54-81.

15 Reid DC, Smith B. Leg length inequality: a review of etiology and management. Physio Can 1984; 36: 177-82.

16 DeLacerda FG, McCrory ML. A case report: effect of a leg length differential on oxygen consumption. J Orthop Sports Phys Ther 1981; 3: 17-20.

17 Gofton JP. Studies in osteoarthritis of the hip. Part IV: Biomechanics and clinical considerations. Can Med Assoc 1971; 104: 1007-11.

18 Gross RH. Leg length discrepancy in marathon runners. Am J Sports Med 1983; 11: 121-4.

19 Blustein SM, D'Amico JC. Limb length discrepancy: identification, clinical significance, and management. I Am Podiatr Med Assoc 1985; 75: 200-6.

20 Brody DM. Running injuries. Clin Symp 1980; 32: 2-36.

21 Friberg $\mathrm{O}$. Leg length asymmetry in stress fractures: a clinical and radiological study. J Sports Med Phys Fitness 1982; 22: 485-8.
22 Sperryn PN, Restan L. Podiatry and the sports physician - an evaluation of orthoses. Br J Sports Med 1983; 17: 129-34.

23 Matheson GO, Clement DB, McKenzie DC, Taunton JE Lloyd-Smith DR, MacIntyre JG. Stress fractures in athletes: a study of 320 cases. Am I Sports Med 1987; 15: 38-57.

24 Mahar RK, Kirby RD, Macleod DA. Simulated leg-length discrepancy: its effect on mean center-of-pressure position and postural sway. Arch Phys Med Rehabil 1985; 86: 822-4.

25 Botte RR. An interpretation of the pronation syndrome and foot types of patients with low back pain. J Am Podiatry Assoc 1981; 71: 243-53.

26 Glles LGF, Taylor JR. Lumbar spine structural changes associated with leg length inequality. Spine 1982; 7: 159-62.

27 Friberg O. Clinical symptoms and biomechanics of lumbar spine and hip joint in leg length inequality. Spine 1983; 8: 643-51.

28 Giles LGF, Taylor JR. Low-back pain associated with leg length inequality. Spine 1981; 6: 510-21.

29 Gofton JP. Persistent low back pain and leg length inequality. I Rheumatol 1985; 12: 747-50.

30 Maquet PGJ. Biomechanics of the knee, 2nd edn. New York: Springer-Verlag, 1984.

31 Solomon L. Patterns of osteoarthritis of the hip. J Bone Joint Surg 1976; 52(B): 175-83.

32 Radin EL, Paul IL, Rose RM. Role of mechanical factors in pathogenesis of primary osteoarthritis. Lancet 1972; i: 519-22.

33 Light LH, McLellan GE, Klenerman L. Skeletal transients on heel strike in normal walking with different footwear. Biomech 1980; 13: 477-80.

34 Voloshin A, Wosk J. Influence of artifical shock absorbers on human gait. Clin Orthop 1981; 160: 52-6.

35 D'Amico JC, Dinowitz HD, Polchaninoff M. Limb length discrepancy: an electrodynographic analsyis. I Am Podiatr Med Assoc 1985; 75: 639-43.

36 Vink $\mathrm{P}$, Huson A. Lumbar back muscle activity during walking with a leg inequality. Acta Morphol Neerl Scand 1988; 25: 261-71. 
competitors. Such power event competitors may demonstrate up to $95 \%$ FT fibres which are metabolically equipped for forceful contractions that depend largely on anaerobic mechanisms for energy supply. FT fibres may also be two to four times faster and approximately $50 \%$ larger than fibres classified as slow. It should be noted here that the athlete's muscle fibre profile is determined both by genetic and environmental (training) factors ${ }^{3}$. No obvious muscle fibre profile differences have been established between men and women competing in similar events.

While muscle size has been associated with muscular strength, hypertrophy (the increase in muscle size or bulk) has been associated with gains in strength. However, because muscular development is partly linked with plasma testosterone levels, men are capable of considerably greater gains in muscle bulk than are women. Some researchers have concluded that muscular hypertrophy in women is not a necessary consequence of strength training.

\section{Conclusion}

Ward and Whipp ${ }^{4}$ plotted the decade-by-decade improvement in the mean running velocity for both men and women for all events between $200 \mathrm{~m}$ and the marathon. It was found that the improvement rate for women is more than double that for men, reflecting both the increased numbers of women coming into sport and the proportionately greater increases in their training.

\section{References ,}

1 Koutedakis Y, Sharp NCC. A modified Wingate test for measuring anaerobic work of the upper body in junior rowers. Br J Sports Med 1986; 20: 153-6.

2 Davies CTM. Strength and mechanical properties of muscle in children and young adults. Scand J Sports Sci 1985; 7: 11-15.

3 Komi PV. The musculoskeletal system. In: Dirix A, Knuttgen HG, Tittel K, eds. The Olympic Book of Sports Medicine. Oxford: Blackwell Scientific Publishers, 1988: 15-39.

4 Ward SA, Whipp BJ. Will women outperform men in Olympic running in the twenty-first century? I Physiol 1990; 423: 24P.

\section{Errata}

Stoneham MD, Morgan NV. Stress fractures of the hip in Royal Marine recruits under training: a retrospective analysis. Br J Sports Med 1991; 25: 145-8.

The publishers wish to apologise for the accidental substitution of an incorrect address for correspondence.

Please send correspondence to:

Surgeon Commander N. V. Morgan RN, Brigade Medical Adviser, 3 Commando Brigade, Stonehouse Barracks, Plymouth, UK.
McCaw ST, Bates BT. Biomechanical implications of mild leg length inequality. Br J Sports Med 1991; 25: 10-13.

The publishers also wish to apologise for the incorrect spelling of Dr S. T. McCaw's name both in the table of contents and the article running head. 